\title{
The role of insulin resistance/hyperinsulinism in the evolution of thyroid nodular disease in humans
}

\begin{abstract}
Thyroid follicular cells are characterized by an intrinsic heterogeneity in responding to various stimuli, regarding their growth potential and function. This heterogeneity appears to be the underlying cause for the focal thyroid cell hyperplasia, under the influence of a growth stimulatory factor, and eventually the development of nodular thyroid disease. Among the main stimulatory factors is the TSH, that regulates both thyroid cell growth and function, and the insulin/IGF system that, through its monogenic effects, can stimulate the proliferation of thyroid cells. The predominance of one or the other thyroid trophic factor, at each time-point, appears to determine the natural history of thyroid nodular disease. Iodine deficiency was, in the past, the main pathogenic factor responsible, through a transient rise in TSH secretion, for the endemic multi nodular goiter with the characteristic colloid thyroid nodules among the inhabitants in the iodine deficient areas of the world. The correction of iodine deficiency was followed by the elimination of the endemic colloid goiter and the emergence of thyroid autoimmunity. The recent epidemic of obesity and metabolic/ insulin resistance syndrome, has been associated with a rise in the incidence of nodular thyroid disease. Characterized by the increase in thyroid gland volume and the formation of hyper plastic nodules. The likely culprit for this association appears to be the hyperinsulinemia that accompanies the insulin resistance syndrome. Thus, thyroid nodular disease appears to have evolved over the years from the endemic colloid goiter to nodular thyroid hyperplasia, in parallel with the changes that have occurred in the thyroid stimulatory factors i.e. from the iodine deficiency, through increased TSH secretion to insulin resistance, through the compensatory hyperinsulinemia.
\end{abstract}

Keywords: insulin resistance, thyroid nodular disease, colloid nodules, hyper plastic nodules, hyperinsulinemia, metabolic syndrome, insulin/igf system
Special Issue - 2018

\author{
Agathocles Tsatsoulis \\ Department of Endocrinology, University of loannina, Greece
}

Correspondence: Agathocles Tsatsoulis, Department of Endocrinology, Emeritus Professor of Medicine/Endocrinology, School of Health Sciences, University of loannina, 45। I0, loannina, Greece, Email atsatsoules@gmail.com

Received: November 30, 2017 | Published: November 15, 2018
Abbreviations: TSH, thyroid stimulating hormone; TSIs, thyroid stimulating immunoglobulin's; IGF, insulin-like growth factor; IGFBP, insulin-like growth factor binding protein; IR, insulin resistance; MS, metabolic syndrome; Pi3 K, phosphatidyl-inositol-3 kinase; MAPK, mitogen -activated protein kinase; US, ultra sound

\section{Introduction}

Nodular thyroid disease or nodular goiter is characterized by the presence of a single or multiple nodules within the thyroid parenchyma. The formation of thyroid nodules is thought to be the result of the intrinsic heterogeneity of thyroid follicular cells, in responding to lowgrade or intermittent stimulus, regarding their growth potential and function. ${ }^{1,2}$ This heterogeneity in growth and function of the thyroid cells appears to be the underlying primary factor for the development of thyroid nodular disease. On the other hand the various secondary factors that stimulate the growth of the thyroid appear to influence the evolution of the thyroid nodular disease. The main thyroid stimulatory factors are the TSH (induced by iodine deficiency, natural goitrogens or inborn errors of thyroid hormone synthesis), thyroid stimulating immunoglobulin's, TSIs (in Graves disease) and thyroid growth stimulating factors ( including insulin, IGF-1 and other growth factors). ${ }^{3}$ It is likely that, when a mild or intermittent stimulus (e.g. mild iodine deficiency, light TSH increase) acts on this heterogeneous group of thyroid follicular cells, only the subpopulation of cells with the highest growth potential will respond, leading to focal thyroid cell hyperplasia. If, on the other hand, the goitrogenic stimulus is strong and persistent (TSIs, in Graves' disease), then the whole population of cells undergo replication resulting in diffuse thyroid hyperplasia. ${ }^{1,2}$ This mini-review highlights the evolution of nodular thyroid disease and the role of insulin resistance/hyperinsulinemia in this phenomenon.

\section{Evolution of thyroid nodular disease: from endemic colloid goiter to nodular thyroid hyperplasia}

Epidemiological evidence and pathophysiology: In the past, iodine deficiency was the main cause of endemic goiter in iodine deficient areas of the world. In response to reduced iodine supply and the associated transient TSH stimulation, the thyroid gland first goes through a period of focal thyroid cell hyperplasia but eventually, because of iodine repletion or decreased requirement for thyroid hormone, the thyroid enters a resting phase characterized by colloid storage and the histological picture of a colloid nodular goiter..$^{3-5}$ The elimination of iodine deficiency by the implementation of iodine prophylaxis programs or, in some countries, through "silent iodine prophylaxis" due to improvement in socioeconomic conditions, was followed by the gradual disappearance of endemic goiter. However, the transition from iodine deficiency to sufficient or excess iodine was associated with the emergence of thyroid autoimmunity. ${ }^{6-8}$ In recent years, the epidemic of obesity and the metabolic/ insulin resistance syndrome has been accompanied by the reappearance of thyroid nodular disease in the form of nodular hyperplasia. ${ }^{9}$ It is believed that insulin resistance is an adaptive mechanism that is activated acutely whenever the organism is in need for increased supply of energy to the brain in the form of glucose. In such cases, like acute 
stressful events or when the organism is fighting off an infection, the activation of insulin resistance temporarily by the action of stress hormones or proinflammatory cytokines, respectively, facilitates the break- down of glycogen to glucose in the liver, to be used for" a fight or flight "response or to fight off the infection. The same adaptive mechanism of insulin resistance is activated on a chronic basis in the current environment in individuals with central obesity as a result of ectopic lipid deposition to peripheral organs and the chronic low-grade inflammation that accompany this condition. ${ }^{10,11}$ It should be noted here that the resistance of target organs to the action of insulin involves only the metabolic effects of insulin and not the mitogenic action on the various tissues of the human body. In fact, insulin resistance is accompanied by compensatory hyperinsulinemia as long as the $\beta$-cells of the pancreas continue to function properly. ${ }^{10}$ In the light of the above, it is apparent that in persons with MS, the underlying insulin resistance and compensatory hyperinsulinemia is associated with increased mitognic effects. Such persons are at high risk for developing manifestations related to the increased mitogenic actions of insulin. Indeed, accumulating evidence suggest that the recent high incidence of thyroid nodules is related to increased prevalence of obesity and insulin resistance. Thus several recent studies have shown an association of insulin resistance with thyroid nodular disease. ${ }^{12}$ These were mainly cross-sectional studies designed to evaluate either the prevalence of insulin resistance in subjects with benign thyroid nodules compared to controls or, alternatively, the prevalence of thyroid nodules in subjects with insulin resistance as evidenced by the measurement of HOMA IR or the presence of MS/type-2 diabetes and in subjects with skin tags, a clinical sign of Hyperinsulinism. The results of the first type of studies have shown that subjects with thyroid nodules have an increased prevalence of insulin resistance and hyperinsulinemia. Consistent with these findings, patients with nodular disease were found to have higher HOMA -IR values compared with individual with a normal thyroid gland. A correlation was found between the nodular size and HOMA IR levels ${ }^{9,12-15}$ the other hand; it was shown that, due to metabolic syndrome, patients with insulin resistance have increased thyroid volume and nodule prevalence. A similar study showed that patients with skin tags, as mark of insulin resistance, have a higher prevalence of US- detected thyroid nodules and larger thyroid glands. ${ }^{15-18}$ Finally a relationship was found between obesity and Hyperinsulinism with increased susceptibility for thyroid nodules in a pediatric population. ${ }^{19}$ Thus, in addition to TSH, insulin is also a thyroid growth factor, which may cause an increase in thyroid volume and the formation of thyroid hyper plastic nodules. Evidence also suggest that IGF-1-dependent and TSH-independent signaling may play a role in growth regulation of the human thyroid gland. This is supported by conditions not associated with increased TSH secretion, like acromegaly, in which high levels of intrathyroid IGF-1 may contribute to thyroid nodular formation..$^{20}$

\section{The Insulin/lgf system and its involvement in thyroid cell hyperplasia}

Molecular aspects: As mentioned earlier, insulin exerts both metabolic and mitogenic effects to target cells. In patients with Metabolic Syndrome and insulin resistance, the later applies only to the metabolic effects of insulin, whereas the mitogenic action is maintained and even exaggerated by the associated hyperinsulinemia. ${ }^{10}$ The IGF system consists of four ligands ( insulin, proinsulin, IGF-1 an IGF-2 ), four receptors ( the insulin receptor, IR, the IGF-1 receptor, the insulin receptor-related receptor and the IGF2 receptor) and six binding proteins (IGFBP-1 to 6). The IR and IGF-1 $\mathrm{R}$ have homologous structure and can heterodimerize leading to the formation of insulin/IGF-1 hybrid receptor. The human IR exists in two isoforms (IR-A and IR-B), generated by alternative splicing of the IR gene with the exclusion (IR-A) or inclusion (IR-B) of 12 amino acids encoded by exon $11 .{ }^{21}$ Insulin and IGFs bind with different affinity to IR and IGF-1R. After ligand binding, phosphorylated receptors activate two main signaling pathways the PI3 K signaling pathway, which mediates metabolic actions and the mitogen-activated protein kinase (MAPK) cascade, which is involved in the regulation of cellular proliferation and gene expression..$^{21}$ The IGF system plays an important role in regulating normal growth and development in the thyroid and appears to be involved in thyroid cell hyperplasia. Functional IR and IGF-1 R are expressed in both cultured thyroid cells and tissue specimens. Also adenoma cell lines synthesize IGF-1, which stimulates cell growth in an autocrine manner. ${ }^{22}$ In patients with insulin resistance, hyperinsulinemia may enhance the bioavailability of IGF-1 and IGF-2 by inhibiting the production of the IGFBP-1 and 2 in the liver. ${ }^{23}$ The increased bioavailability of IGFs may contribute to thyroid cell proliferation acting through the IGF-1R or the IR itself. Yet, hyperinsulinemia, by directly activating the IR-A, may favor its non-metabolic actions and the induction of the pro-mitogenic MAPK cascade. ${ }^{21}$ With the above in mind, it is suggested that, in patients with insulin resistance, the concomitance of the increased activity of the IGF axis and hyperinsulinemia may explain the increase in the prevalence of thyroid nodular disease.

\section{Conclusion}

Recent evidence suggests a role of insulin resistance/ Hyperinsulinism in the evolution of thyroid nodular disease. This involves the transition from endemic colloid goiter, due to iodine deficiency and the associated transient increase in TSH secretion, to the increase in the incidence of nodular thyroid hyperplasia, due to hyperinsulinemia, as a manifestation of the current epidemic of the insulin resistance syndrome. In the interim, the elimination of iodine deficiency was followed by the disappearance of endemic nodular goiter and the emergence of thyroid autoimmunity, as a result of the increase in iodine intake. The changing histological phenotype of nodular thyroid disease i.e. from colloid to hyper plastic nodular goiter, is mainly due to changes in the thyroid stimulatory factors acting on thyroid cells that are characterized by an intrinsic heterogeneity regarding their growth potential and function. Thus, in the case of colloid goiter, the stimulus is the transient increase in TSH secretion as a result of iodine deficiency. The initial rise in TSH appears to be responsible for the focal thyroid hyperplasia. This is followed by a resting phase characterized by colloid storage resulting in the formation of colloid nodules. On the other hand, the hyperinsulinemia constitutes a mild but continuous stimulus for thyroid cell proliferation, leading to increase in thyroid gland volume and the formation of hyper plastic nodules. It is concluded that the current increase in the incidence of nodular thyroid disease in humans is another manifestation of the metabolic/insulin résistance syndrome. It is likely that the compensatory hyperinsulinemia, through its mitogenic effects, is responsible for the re-emergence of nodular thyroid disease.

\section{Acknowledgements}

There is nothing to declare regarding this article. 


\section{Conflict of interest}

The author declares no conflict of interest.

\section{References}

1. Studer H, Derwahl M. Mechanisms of non-neoplastic endocrine hyperplasia a changing concept: A review focused on the thyroid gland. Endocr Rev. 1995;16(4):411-426.

2. Derwahl M. Molecular aspects of the pathogenesis of nodular goiters, thyroid nodules and adenomas. Exp Clin Endocrinol Diabetes. 1996;104(4):32-35.

3. Kimura T, Van Keymeulen A, Golstein J, et al. Regulation of thyroid cell proliferation by TSH and other factors: a critical evaluate on off in vitro models. Endocr Rev. 2001;22(5):631-656.

4. NIlsson M. Iodine handling by the thyroid epithelial cell. Exp Clin Endocrinol diabetes. 2001;109(1):13-17.

5. Gharib H, Papini E. Thyroid nodules: clinical importance assessment and treatment. Endocrinol Metab Clin North Am. 2007;36(3):707-773.

6. Laurberg P, Pedesren KM, Vestergaard H, et al. High incidence of multinodular goiter in the elderly population in a low iodine intake area vs high incidence of Graves' disease in the young in a high iodine intake area: comparative surveys of thyrotoxicosis epidemiology in EastJutland Denmark and Iceland. J Intern Med. 1991;229(5):415-420.

7. Tsatsoulis A, Johhnson EO, Andrikoula M, et al. Thyroid autoimmunity is associated with higher urinary iodine concentrations in an iodinedeficient area of North Western Greece. Thyroid. 1999;9(3):279-283.

8. Zois C, Stavrou I, Kalogera C, et al. High prevalence of autoimmune thyroiditis in school children after elimination of iodine deficiency in North Western Greece. Thyroid. 2003;13(5):485-489.

9. Ayturk S, Gursoy A, Kut A, et al. Metabolic syndrome and its components are associated with increased thyroid volume and nodule prevalence in a mild to moderate iodine-deficient area. Eur J Endocrinol. 2009;161(4):599-605.

10. Eckel RH, Grundy SM, Zimmet PZ. The Metabolic Syndrome. Lancet. 2005;365(9468):1415-1428.
11. Tsatsoulis A, Mantzaris MD, Bellou S, et al. Insulin resistance: an adaptive mechanism becomes maladaptive in the current environmentan evolutionary perspective. Metabolism. 2013;62(5):622-633.

12. Heidari Z, Mashhadi A, Nosratzehi S. Insulin resistance in patients with benign thyroid nodules. Arch Iran Med. 2015;18(9):572-576.

13. El Saghier EOA, Hassan ZAB, Shawky AMA. Euthyroid nodular disease in relation to Insulin resistance. Int J Diabetes Res. 2015;4(3):49-57.

14. Reszzonico J, Rezzonico M, Pusiol E, et al. Introducing the thyroid gland as another victim of the insulin resistance syndrome. Thyroid. 2008;18(4):461-464.

15. Yasar HY, Ertugrul O, Ertugrul B, et al. Insulin resistance in nodular thyroid disease. Endocr Res. 2011;36(4):167-174.

16. Medeiros De Sousa PA, Vaisman M, Carneiro JR, et al. Prevalence of goiter and thyroid nodular disease in patients with class III obesity. Arq Bras Endocrinol Metabol. 2013;57(2):120-125.

17. Rezzonico J, Rezzonico M, Pusiol E, et al. High prevalence of thyroid nodules in patients with acrochordons (skin tags). Possible role of insulin resistance. Medicina (B Aires). 2009;69(3):302-304.

18. Anil C, Akkurt S, Ayturk S, et al. Impaired glucose metabolism is a risk factor for increased thyroid volume and nodule prevalence in a mild to moderate iodine deficient area. Metabolism. 2013;62(7):970-975.

19. Javier C, Ricardo P, Hugo C, et al. To assess the relationship between hypeinsulinism and thyroid nodules in children with overweight and obesity. Endocrinol Metab Synn. 2015;4:185.

20. C Heun NW, Boyages SC. The thyroid gland in acromegaly: An ulltrasonographic study. Clin Endocrinol. 1997;46(5):545-549.

21. Malaquarnera R, Morcavallo A, BelfioreA. The insulin and IGF-I pathway in endocrine glands carcinogenesis. J Oncol. 2012;2012:635614.

22. Malaquarnera F, Frasca A, Garozzo F, et al. Insulin receptor is forms and insulin -like growth factor receptor in human follicular cell precursors from papillary thyroid cancer and normal thyroid. J Clin Endocrinol Metab. 2011;96(3):766-774.

23. Baxter RC, Dayson JM, Turtle JR. Somatogenic receptors in rat liver: regulation by insulin. Endocrinol. 1984;107(14):1176-1181. 